\title{
A study of innate immunity in patients with end-stage renal disease: Special reference to toll-like receptor- 2 and -4 expression in peripheral blood monocytes of hemodialysis patients
}

\author{
YOSHIKAZU KUROKI, KENJI TSUCHIDA, ISHUN GO, MASATO AOYAMA, \\ TOSHIHIDE NAGANUMA, YOSHIAKI TAKEMOTO and TATSUYA NAKATANI \\ Department of Urology and Division of Artificial Kidney, \\ Osaka City University Graduate School of Medicine, Osaka, Japan
}

Received December 18, 2006; Accepted January 25, 2007

\begin{abstract}
It was recently shown that toll-like receptors (TLR) play a critical role in innate immunity. However, no study has been conducted on TLR expression in hemodialysis (HD) patients. The present study was undertaken to examine innate immunity and the roles played by endotoxins (ET) contained in dialysate in HD patients, by analysis of TLR expression and reactivity. TLR-2 and TLR-4 expression on monocytes was investigated by flow cytometry in the following groups of subjects: healthy controls, patients on HD, patients with end-stage renal disease (ESRD) and patients on peritoneal dialysis (PD). The expression of TLRs on monocytes under stimulation with lipopolysaccharide was also investigated. Expression of TLR-4 was lower in the HD group than in the healthy controls $(\mathrm{p}<0.05)$, while expression of TLR-2 was lower in the PD group than in the healthy controls $(\mathrm{p}<0.05)$. As the duration of dialysis became longer, TLR-4 expression decreased $(p<0.01)$. TLR-2 was not correlated with duration of dialysis, and the magnitude of decrease in TLR-4 expression following stimulation with ET became smaller $(p=0.0006)$. Suppression of expression of TLR-4 was noted in HD patients, and TLR-4 expression was reduced as the duration of dialysis became longer. Reduced TLR-4 expression may be associated with the compromised immune function in HD patients. It seems possible that chronic stimulation with ET suppresses the expression of TLR-4.
\end{abstract}

\section{Introduction}

In Japan, the number of patients with end-stage renal disease who required dialysis was $>250,000$ as of December, 2005 . Of these patients, $95 \%$ are being treated with hemodialysis

Correspondence to: Dr Yoshikazu Kuroki, Department of Urology and Division of Artificial Kidney, Osaka City University Graduate School of Medicine, 1-5-7 Asahi-machi, Abeno-ku, Osaka 5458586, Japan

E-mail: m4456591@msic.med.osaka-cu.ac.jp

Key words: toll-like receptor, hemodialysis, immunity
(HD). Infection is the second leading cause of death among hemodialysis patients, and infection tends to be severe among these patients (1). It has been shown by clinical data that the prevalence of tuberculosis is high among hemodialysis patients and that infections such as flu syndrome among hemodialysis patients are difficult to cure and tend to persist and follow a severe course, and that the incidence of malignant tumors is higher among hemodialysis patients than among individuals with intact renal function (2). Factors possibly responsible for the reduced immunity and increased susceptibility to infection in hemodialysis patients include uremia, malnutrition, renal anemia, and various others.

A dialyzer and dialysate are required for hemodialysis. The dialysate is susceptible to contamination by aquatic flora such as gram-negative bacilli, and adequate care is needed to prevent contamination by endotoxins (ET) contained in such microorganisms. However, since the dialysate is sometimes contaminated not only by gram-negative bacilli but also by gram-positive cocci and fungi, it can be contaminated not only by ET but also by peptidoglycans (PG), muramyldipeptide, ß-D-glucan, teichoic acid, and other agents (3).

Recently, high flux membranes have often been used to eliminate proteins of low molecular weight and uremic substances of medium to high molecular weight. However, it has been suggested that, due to back diffusion of dialysate or back filtration, hazardous substances such as ET contained in the dialysate can invade the body or react with it via the dialysis membrane. The ET adsorbed onto the dialysis membrane can thereby act on peripheral blood monocytes and stimulate the production of inflammatory cytokines even when it does not directly invade the body, and the peripheral blood mononuclear cells of hemodialysis patients with a contaminated dialysate exhibit reduced reactivity to ET or PG $(4,5)$.

Clinically, it is expected that if the dialysate can be kept clean to minimize contamination by ET, it will be possible to reduce chronic inflammation due to dialysis and thereby prevent many complications associated with hemodialysis (6). In fact, it has been reported that keeping the dialysate clean alleviated renal anemia and reduced the incidences of dialysis-related amyloidosis and other conditions $(7,8)$. However, many questions remain concerning the mechanisms responsible for these changes. 
It was recently shown that a group of receptors called toll-like receptors (TLRs) are required for recognition of pathogens and subsequent immune reactions to them. To date, 10 TLRs have been identified. TLR-2 recognizes peptidoglycans (PG), which are components of the cell wall of gram-positive bacteria (they are also contained in gramnegative bacteria, though in smaller amounts). TLR-2 also recognizes bacterial lipoproteins, and TLR-4 recognizes lipopolysaccharides (LPS, a type of ET) (9-11). When TLR recognizes a microbial component, it activates an intracellular signal transduction pathway and stimulates the transfer of $\mathrm{NF}-\kappa \mathrm{B}$ (a transcription factor) into the nucleus via the common adapter molecule MyD88, leading to production of various inflammatory cytokines and expression of costimulatory molecules (12).

However, no study has been conducted on TLR expression on the peripheral blood monocytes of patients in end-stage renal disease or those undergoing hemodialysis. The present study was undertaken to examine innate immunity and the roles played by ET contained in dialysate in dialysis patients, by analysis of TLR expression and reactivity.

\section{Materials and methods}

Isolation of peripheral blood mononuclear cells (PBMC) and determination of expression of TLR-2 and TLR-4. Heparinized peripheral blood was collected in a volume of $15 \mathrm{ml}$. The regional ethics committee approved the study protocol, and informed consent was obtained from each subject. Blood samples from HD patients were obtained before hemodialysis. Patients with a history of malignancy were excluded. Exclusion criteria also included antibiotic or immunomodulatory drug use and C-reactive protein positivity. PBMC were isolated from whole blood on the Ficoll-Paque (Axis-Shield, Oslo, Norway) gradient. The combination of this $15-\mathrm{ml}$ portion of blood and $15 \mathrm{ml}$ of physiological saline was placed in a Lymphoprep tube (Axis-Shield), which was agitated by turning it upside-down. It was then centrifuged for $20 \mathrm{~min}$ ( $800 \mathrm{x} \mathrm{g}$ at room temperature). The PBMC fraction was harvested and re-suspended in phosphate-buffered saline (PBS) at a concentration of $1 \times 10^{7}$ cells $/ \mathrm{ml}$.

A $100-\mu 1\left(1 \times 10^{6}\right.$ cells $)$ portion of the suspension was stained with phycoerythrin (PE)-negative control antibody (Dako, Denmark), PE-labeled anti-TLR-2 antibody (TL2.1; Imgenex, San Diego, CA), and PE-labeled anti-TLR-4 antibody (HTA125; Imgenex), followed by incubation at $4{ }^{\circ} \mathrm{C}$ for $30 \mathrm{~min}$. After incubation, each sample was cleansed with phosphate buffer with $1 \%$ bovine serum albumin (1\% BSA PBS). The cleansing solution was removed and $1 \%$ paraformaldehyde PBS (500 $\mu \mathrm{l})$ was added. The cells were finally passed through a mesh, and flow cytometry was performed using a FACSCaliber (Becton Dickinson, San Diego, CA). Monocytes were gated with a forward and sidescatter plot pattern and MFI (mean fluorescence intensity) was measured. The analysis was performed on Cell Quest software (Becton Dickinson).

TLR-2 and TLR-4 expression. TLR-2 and TLR-4 expression on monocytes was investigated in the following groups of subjects: i) healthy controls and patients on hemodialysis
(HD); ii) HD patients with end-stage renal disease (ESRD), and patients on peritoneal dialysis (PD); iii) patients on HD using different dialyzers: comparison of polysulfone (PS) membrane users with users of other membranes; and iv) relationship of TLR-2 and TLR-4 expression to duration of dialysis in HD patients.

TLR-2 and TLR-4 expression following stimulation with LPS. The PBMC suspension was incubated with LPS, followed by washing with PBS. LPS from Escherichia coli O111:B4 was purchased from Chemicon International (Temecula, CA). After staining with PE-labeled anti-TLR-2 antibody and PElabeled anti-TLR-4 antibody, the suspension was additionally incubated for $30 \mathrm{~min}$ at $4^{\circ} \mathrm{C}$. After incubation, the sample was washed with $1 \%$ BSA PBS. The cleansing fluid was removed, and $1 \%$ paraformaldehyde PBS (500 $\mu 1)$ was added. The cells were then passed through a mesh, and subjected to flow cytometry with a FACSCaliber to measure MFI. Analysis was performed with Cell Quest software. The experiment was performed using varying concentrations of LPS added to the PBMC as well as varying durations of incubation $(n=5)$. Five durations of incubation $(0,15,30,60$, and $120 \mathrm{~min}$ ) were tested at an LPS concentration of $200 \mathrm{pg} /$ $\mathrm{ml}$. Furthermore, five concentrations of LPS $(0,20 \mathrm{pg} / \mathrm{ml}$, $200 \mathrm{pg} / \mathrm{ml}, 2000 \mathrm{pg} / \mathrm{ml}$, and $20 \mathrm{ng} / \mathrm{ml}$ ) were tested, with the incubation time set at $2 \mathrm{~h}$ for each LPS level $(\mathrm{n}=12)$. The expression of TLR-2 and TLR-4 at these varying conditions was determined to identify the optimal set of conditions for stimulation of such expression.

The expression of TLR-2 and TLR-4 in peripheral blood monocytes under stimulation with LPS was investigated in the following groups under the optimal conditions identified in the above described experiment: i) healthy individuals and HD patients; ii) patients on HD using different dialyzers (comparison of PS membrane users with users of other membranes); and iii) relationship of TLR-2 and TLR-4 expression to the duration of dialysis in HD patients. Changes in fluorescence intensity following stimulation with LPS were expressed as percentages relative to prestimulation intensity $(=100 \%)$.

Statistical analysis. The Mann-Whitney U test was employed for statistical comparison among groups. Pearson's coefficient of correlation was used for analysis of TLR-2 and TLR-4 expression to duration of dialysis in HD patients. Findings of $\mathrm{p}<0.05$ were considered significant.

\section{Results}

The background variables in each group (male-to-female ratio, age, duration of dialysis, and genesis of renal failure) are summarized in Table I. An example of the histogram used in this study is shown in Fig. 1.

\section{TLR-2 and TLR-4 expression}

Healthy controls and HD patients (Fig. 2). Expression of TLR-2 on peripheral blood monocytes was slightly stronger in HD patients $(64.7 \pm 18.7)$ than in healthy controls $(52.3 \pm 14.9)$, although the difference between groups was not statistically significant. Expression of TLR-4 was signifi- 
Table I. Patient medical histories.

\begin{tabular}{lcccc}
\hline & HD patients & PD patients & Control & ESRD patients \\
\hline Number (n) & 31 & 9 & 10 & 10 \\
Male/female (n) & $11 / 20$ & $6 / 3$ & $4 / 6$ & $8 / 2$ \\
Age (year) & $65.9 \pm 9.33$ & $61.3 \pm 8.83$ & $32.9 \pm 4.63$ & $62.3 \pm 12.2$ \\
HD duration (year) & $11.0 \pm 6.46$ & $3.66 \pm 2.34$ & - & - \\
Dialysis membrane (polysulfone/non-PS) & $15 / 16$ & - & - & - \\
Genesis of renal failure (DM/non-DM) & $0 / 31$ & $2 / 7$ & - & $4.02 \pm 2.65$ \\
Serum creatinine (mg/dl) & $9.08 \pm 1.79$ & $9.43 \pm 4.33$ & - & \\
\hline
\end{tabular}
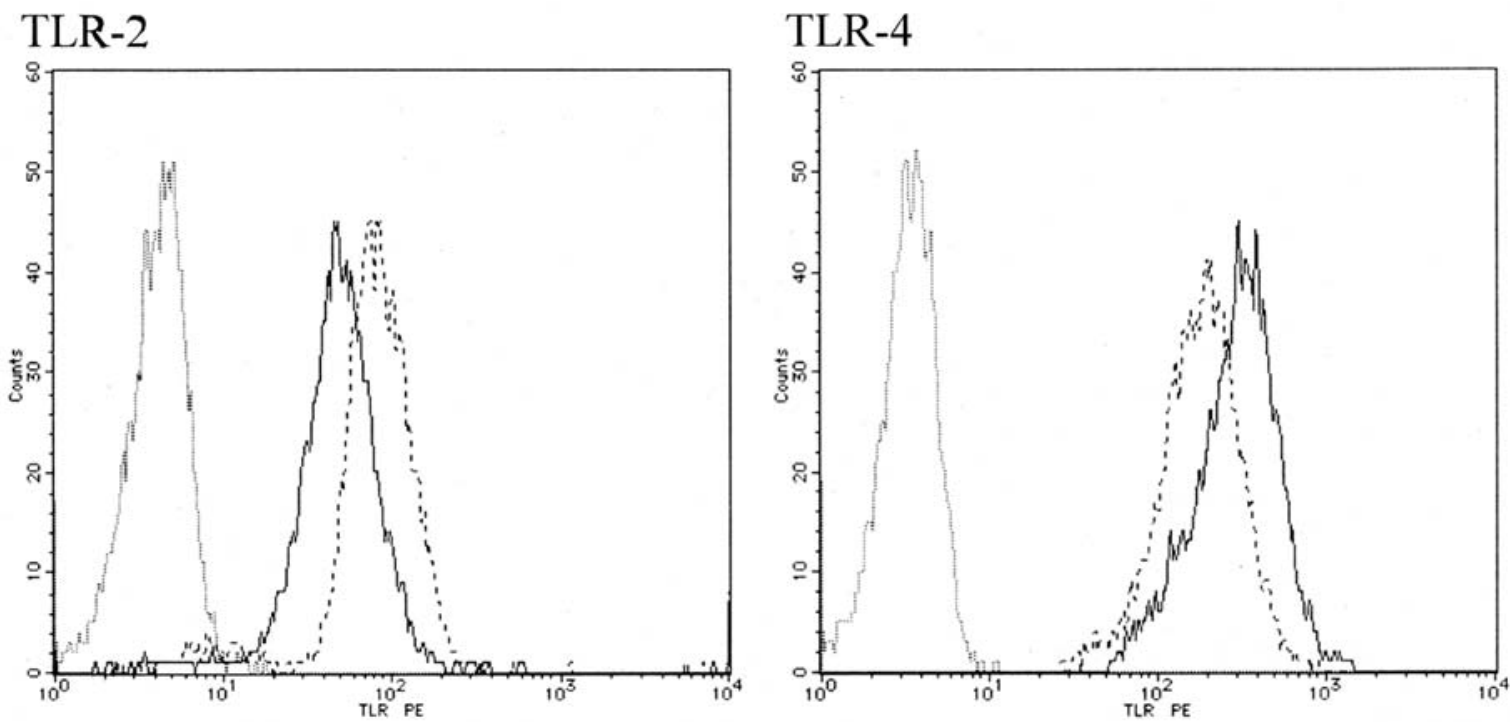

Figure 1. Monocyte surface expression of TLR-2 and TLR-4. Flow cytometry linear-log scale histogram with fluorescence intensity on x-axis plotted against cell counts. Result of one representative HD patient is shown. The gray line represents expression of the negative control; the solid line, the basal expression and the dashed line, the LPS-stimulated sample (15-min incubation with $200 \mathrm{pg} / \mathrm{ml}$ ).

\section{TLR-2 expression}

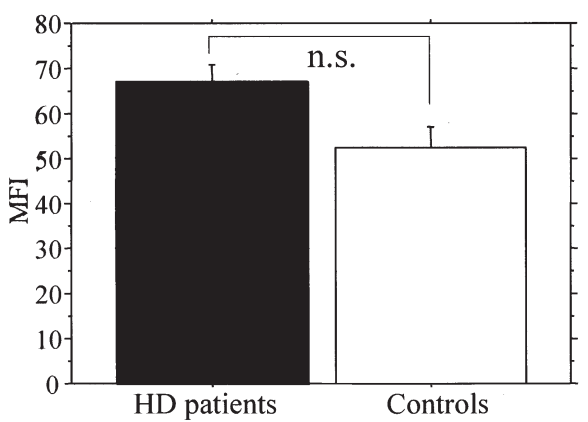

\section{TLR-4 expression}

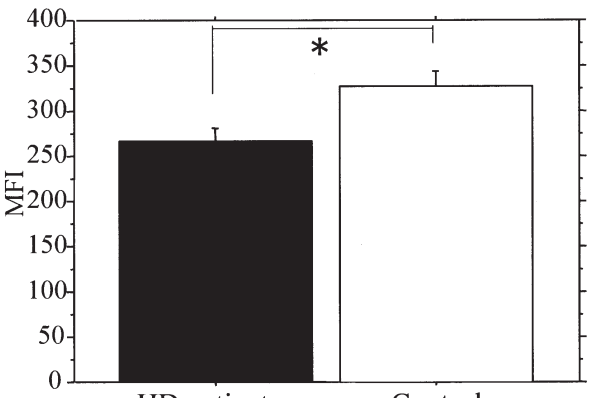

HD patients

Controls

Figure 2. Comparison of TLR-2 and TLR-4 expression on monocytes in control subjects ( $\mathrm{n}=10)$ and HD patients ( $\mathrm{n}=31$ ). PBMC from controls and HD patients were stained with PE-conjugated anti-TLR-2 and PE-conjugated TLR-4 mAb. MFI of monocyte TLR-2 and TLR-4 expression is shown. TLR-4 expression was significantly decreased in HD patient. Data are presented as means $\pm \mathrm{SD} ;{ }^{*} \mathrm{p}<0.05$.

cantly lower in the HD group $(266.4 \pm 88.0)$ than in the healthy controls $(327.9 \pm 51.8)(\mathrm{p}<0.05)$.

HD, ERSD and PD groups (Fig. 3). Expression of TLR-2 on peripheral blood monocytes was significantly lower in the PD group $(36.8 \pm 15.7)$ than in the HD group $(64.7 \pm 18.7)$ and
ESRD group $(50.5 \pm 22.2)$. Expression of TLR-2 was significantly lower in the PD group than in the healthy controls $(\mathrm{p}<0.05)$. Expression of TLR-4 tended to be lower in the HD group (266.4 \pm 88.0$)$ than in the PD group (281.2 \pm 57.3$)$ and ESRD group (307.3 \pm 64.7$)$, although the differences between groups were not statistically significant. 

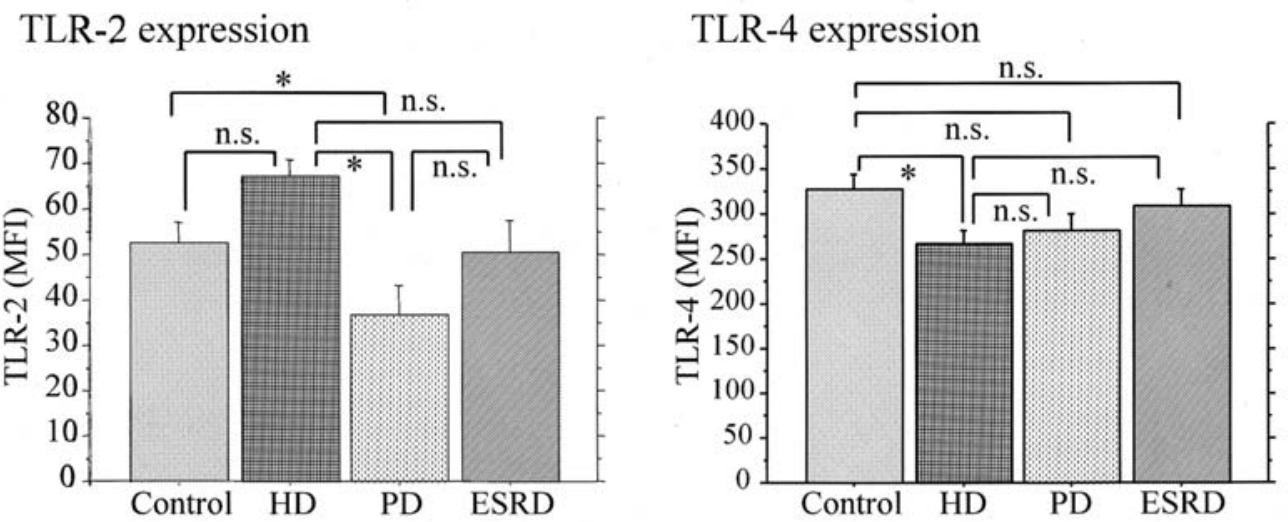

Figure 3. Comparison of TLR-2 and TLR-4 expression on monocytes in control subjects $(n=10)$, ESRD ( $n=10)$, PD ( $n=9)$ and HD patients $(n=31)$. MFI of monocyte TLR-2 and -4 expression is shown. TLR-2 expression was significantly decreased in PD patients (versus control and HD). TLR-4 expression was significantly decreased in HD patients (versus control). Data are presented as means $\pm \mathrm{SD} ;{ }^{*} \mathrm{p}<0.05$.

\section{TLR-2 expression}

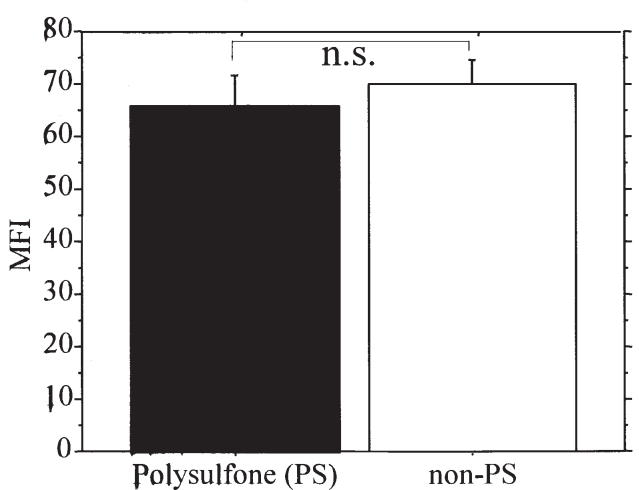

\section{TLR-4 expression}

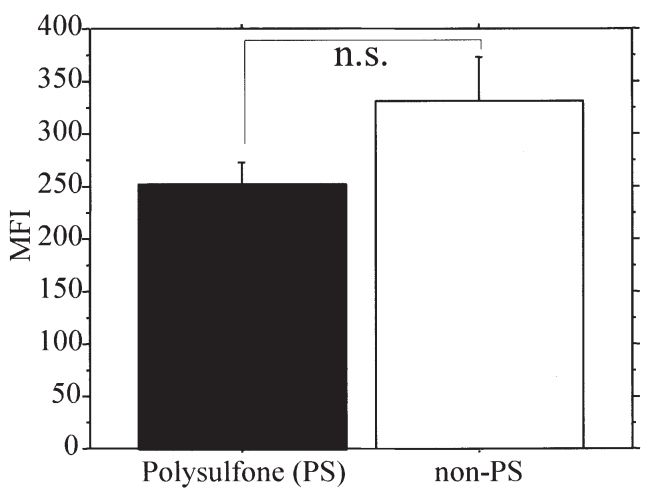

Figure 4. Comparison of TLR-2 and TLR-4 expression on monocytes in two dialysis membranes (polysulfone, n=15; non-PS, n=16). MFI of monocyte TLR-2 and TLR-4 expression is shown. TLR-4 expression tended to decrease in PS patients. Data are presented as means \pm SD.

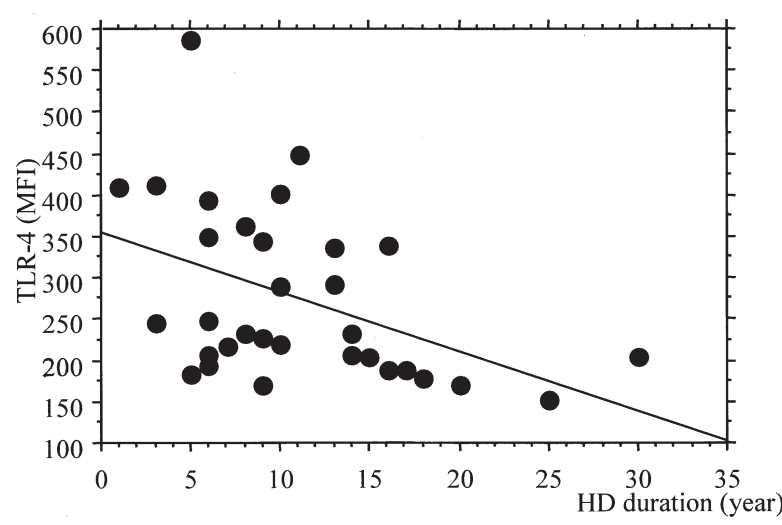

Figure 5. Correlation between the monocyte surface expression of TLR-4 and HD duration. $\mathrm{R}=-0.447, \mathrm{p}=0.0103$ (Pearson's correlation coefficient was used for measurement and $\mathrm{p}<0.05$ was regarded as a statistically significant difference).

Patients on HD using different dialyzers: comparison of PS membrane users with users of other membranes (Fig. 4). There were no statistically significant differences in TLR-4 or TLR-2 expression between PS membrane users and users of other membranes. However, expression of TLR-4 tended to be lower in the PS membrane users.

Relationship of TLR-2 and TLR-4 expression to duration of dialysis in $H D$ patients. As the duration of dialysis became longer, TLR-4 expression decreased significantly $(\mathrm{p}<0.01)$ (Fig. 5), while expression of TLR-2 was not correlated with duration of dialysis (data not shown).

\section{TLR-2 and TLR-4 expression under stimulation with LPS}

Determination of optimal conditions of stimulation (Fig. 6). Expression of TLR-2 was significantly stronger in the presence of 20 or $200 \mathrm{pg} / \mathrm{ml}$ LPS than in the absence of LPS. Expression of TLR-4 was significantly weaker in the presence of 20 or $200 \mathrm{pg} / \mathrm{ml}$ than in the absence of LPS. The optimal concentration of LPS was therefore set at $200 \mathrm{pg} / \mathrm{ml}$, since adjustment was then easy and errors appeared to be smaller. The optimal duration of incubation in the presence of LPS was set at $15 \mathrm{~min}$, since TLR-4 expression was significantly lower than baseline after $15 \mathrm{~min}$ of incubation.

Healthy controls and HD patients. In both groups, expression of TLR-2 increased significantly and expression of TLR-4 


\section{TLR-2}
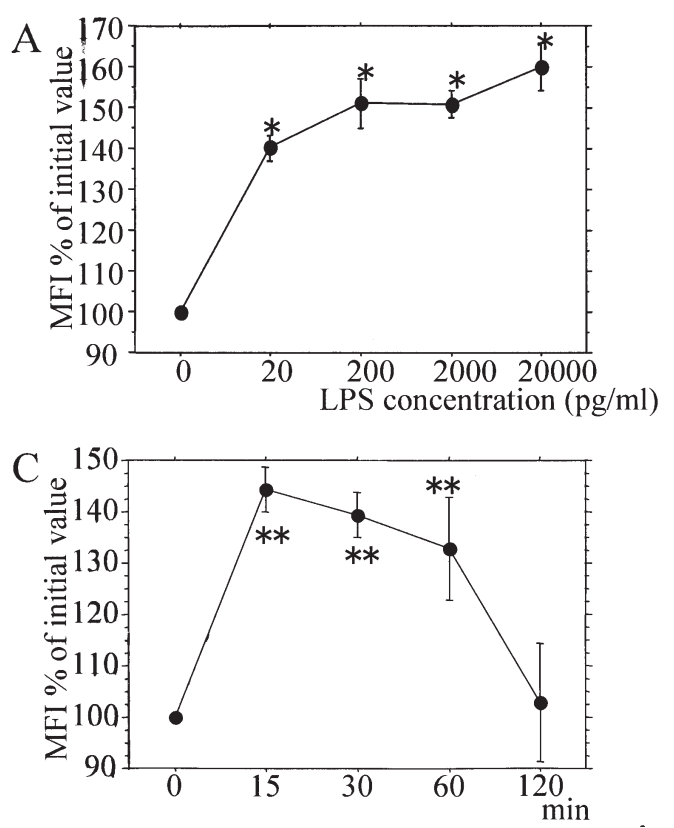

TLR-4

B
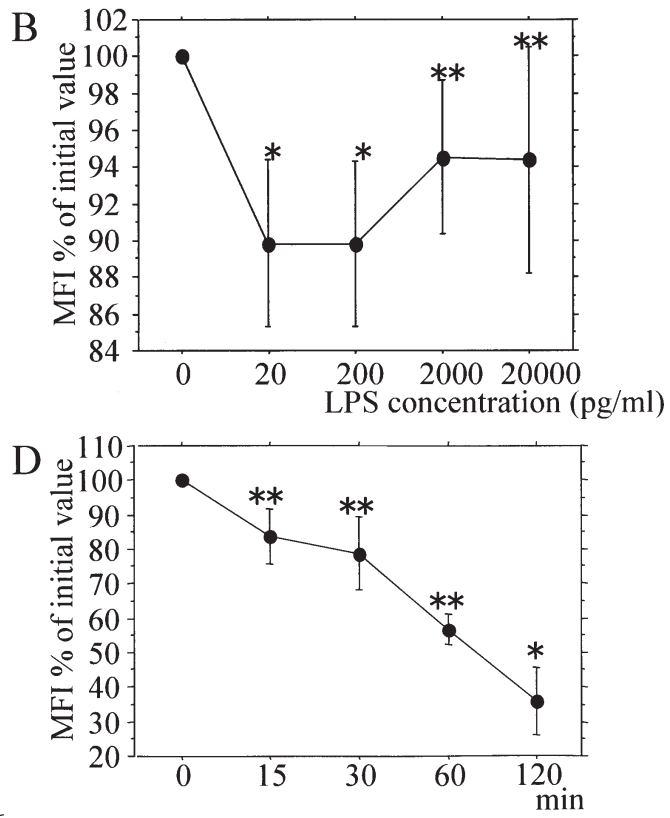

Figure 6. Effect of LPS on monocyte surface expression of TLR-2 and TLR-4. (A,B) Isolated PBMC from healthy controls (n=12) were incubated with the indicated concentrations of LPS for $15 \mathrm{~min}$. (C,D) Isolated PBMC from healthy controls ( $\mathrm{n}=5$ ) were incubated with $200 \mathrm{pg} / \mathrm{ml}$ of LPS for the indicated periods. Data are presented as means $\pm \mathrm{SD} .{ }^{*} \mathrm{p}<0.01$ and ${ }^{* *} \mathrm{p}<0.05$ compared with the value at $0 \mathrm{~min}$ or $0 \mathrm{pg} / \mathrm{ml}$ of LPS.

TLR-2
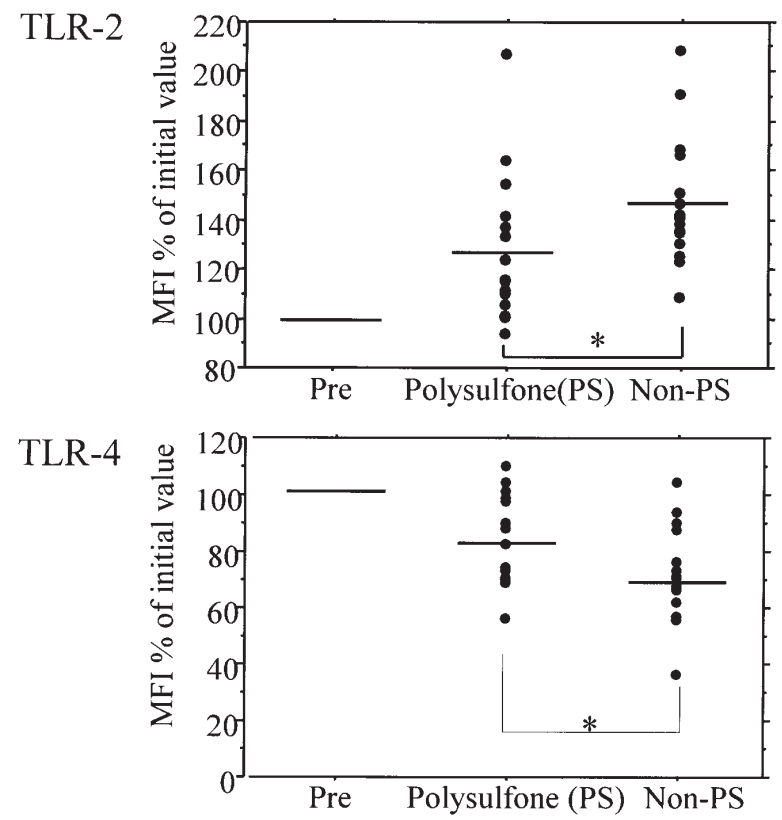

Figure 7. Comparison of monocyte surface expression of TLR-2 and TLR-4 after LPS stimulation as measured by flow cytometry in two membranes (polysulfone, $\mathrm{n}=15$; non-PS, $\mathrm{n}=16$ ). Data are presented as individual MFI values as a percentage of the pre-stimulation value. Horizontal bars represent median values; ${ }^{*} \mathrm{p}<0.05$.

decreased significantly following stimulation with LPS. The magnitude of increase in TLR-2 expression was greater in the HD group than in the healthy group, and the magnitude of decrease in TLR-4 expression was smaller in the HD group than in the healthy group, although none of these differences were statistically significant (data not shown).
Patients on HD using different dialyzers (Fig. 7). The PS membrane users had significantly higher TLR-2 expression following stimulation with LPS than the users of other membranes $(\mathrm{p}<0.05)$. The decrease in TLR-4 expression following stimulation with LPS was significantly smaller in magnitude for PS membrane users $(\mathrm{p}<0.05)$.

Relationship to history of dialysis in HD patients. As the duration of dialysis increased, the magnitude of decrease in TLR-4 expression following stimulation with LPS became significantly smaller ( $\mathrm{p}=0.0006$ ) (Fig. 9), while the increase in TLR-2 expression following LPS stimulation became significantly smaller ( $\mathrm{p}=0.0095)$ (Fig. 8).

\section{Discussion}

The mammalian toll molecules and a toll-like receptor (TLR) family have recently been discovered. The roles played by these molecules in innate immunity have gradually been unveiled. TLRs induce host-defensive reactions mediated by the formation of antimicrobial peptides, in front-line defenders such as epithelial cells (13). TLRs expressed on epithelial cells and macrophages induce the production of cytokines, leading to rapid induction of defensive reactions primarily involving neutrophils. TLRs expressed on dendritic cells activate them and induce adaptive immune responses through activation of $\mathrm{T}$ lymphocytes. Recognition of invading pathogens by TLRs and transmission of activation signals are directly linked to the decision regarding the need for eliminating a given pathogen from the body. TLRs play important roles in the decision regarding the need for immune responses (12).

It has been reported that susceptibility to infection is high when TLRs are deficient. Among the isoforms of TLRs, 
TLR-2

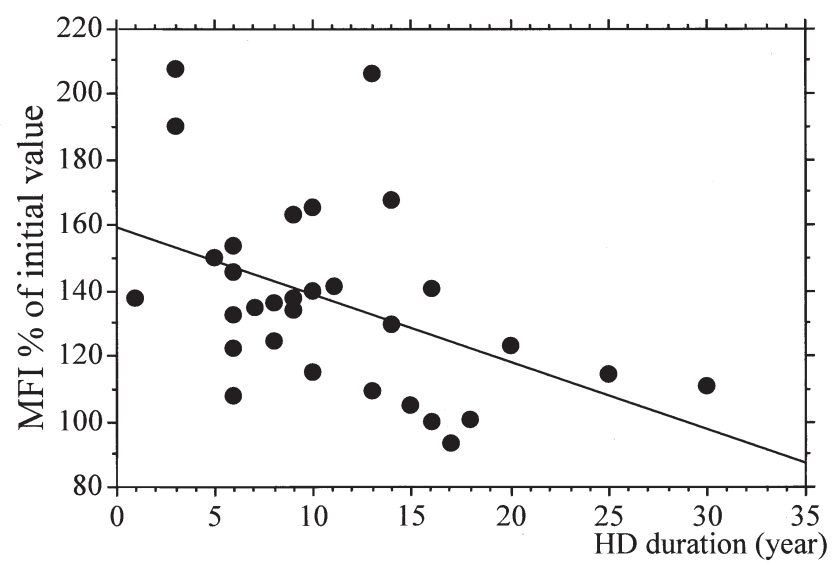

Figure 8. Correlation between the monocyte surface expression of TLR-2 after LPS stimulation and HD duration. Data are represented as individual MFI values as a percentage of the pre-stimulation value. $R=0.458, p=0.0095$ (Pearson's correlation coefficient was used for measurement and $\mathrm{p}<0.05$ was regarded as a statistically significant difference).

\section{TLR-4}

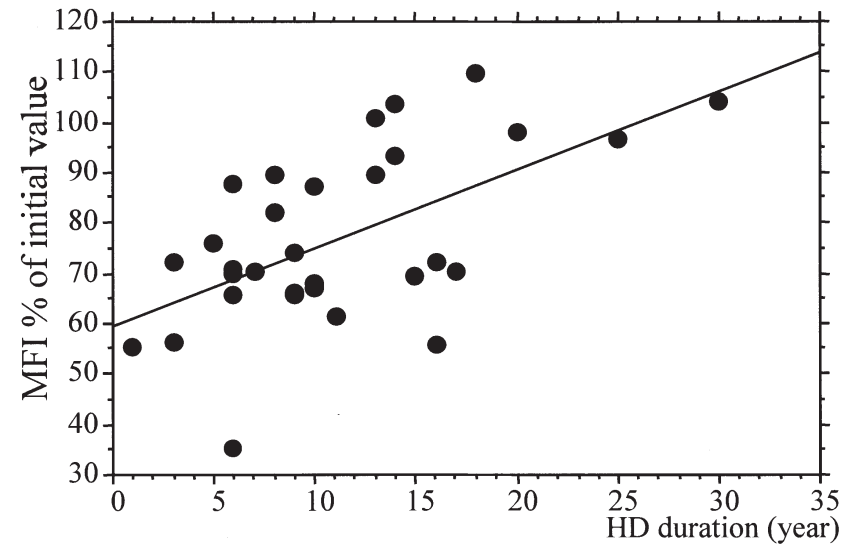

Figure 9. Correlation between the monocyte surface expression of TLR-4 after LPS stimulation and HD duration. Data are presented as individual MFI values as a percentage of the pre-stimulation value. $\mathrm{R}=0.582$, $\mathrm{p}=0.0006$, (Pearson's correlation coefficient was used for measurement and $\mathrm{p}<0.05$ was regarded as a statistically significant difference).

TLR-4 and TLR-2 play particularly important roles in defense against infection. TLR-2 forms a heterodimer with TLR-1 or TLR-6 to recognize the PG or polypeptides of gram-positive bacteria (14). TLR-4 binds to a molecule called MD-2 in the extracellular domain, and the resultant TLR-4MD-2 complex recognizes LPS, the lipid constituting the tunica externa of gram-negative bacteria (15). Failure of TLR-2 or TLR-4 to recognize pathogens markedly reduces immune responses to them. For example, it has been reported that deficiency of at least one of TLR-4 or MD-2 resulted in a high degree of susceptibility of mice to gram-negative bacterial infection $(11,16)$. Mutation of TLR-4 is often observed in patients infected with gram-negative bacteria admitted to the intensive care unit $(17,18)$. No such finding has been obtained for CD14. TLR-2-deficient mice have been found to exhibit a high degree of susceptibility to infection with gram-positive bacteria (19). TLR-2 gene mutation has also been reported in humans, suggesting the possibility of a correlation between this mutation and gram-positive bacterial infection (20). Associations of TLR-4 and TLR-2 with other infections have also been reported (21-23).

It has been reported that patients on dialysis usually exhibit compromised immunity and high susceptibility to infection. As a result, infection is the second leading cause of death among dialysis patients (1). Reports have been published concerning reduced $\mathrm{T}$ cell function and compromised humoral immunity (primarily involving B cells) in dialysis patients $(24,25)$. Although these abnormalities have been suggested to involve uremia-associated substances, malnutrition, renal anemia, and other factors, the exact reasons remain unknown. HD patients, for whom dialysate is used, have the risk of exposure to ET contained in the dialysate. Peritonitis is the most serious complication in PD patients. Since peritonitis is often caused by gram-positive bacteria, it can expose the patient to PG. It appears possible that daily exposure to these components of bacteria reduces the immunity of dialysis patients. Various reports have been published concerning the formation of inflammatory cytokines by PBMC in dialysis patients. Tsuchida et al reported the interesting finding that reaction of PBMC to ET and PG is probably suppressed in patients undergoing hemodialysis using contaminated dialysate (3). However, no study has been published concerning TLR in dialysis patients. The present study was therefore undertaken to examine TLR expression and reactivity, with the goals of investigating the effects of the microbial components (ET, etc.) in dialysate on the body as well as their effects on innate immunity in dialysis patients.

In the present study, TLR-4 expression was significantly lower in the HD group than in the healthy group. In connection with this observation, Ikushima et al reported a study in which blood was sampled from 83 patients before and immediately, 4 h, 24 h, 3 days, and 7 days after gastrointestinal surgery to check for changes in TLR-2 and TLR-4 expression on monocytes. They reported that TLR-2 and TLR-4 expression was lowest 3 days and 1 day after surgery, respectively. They interpreted these results as indicating that stress due to gastrointestinal surgery reduced the expression of TLR-2 and TLR-4, and that this reduction in TLR-2 and TLR-4 expression plays a role in reducing resistance to infection and immune function (26). Reduction of expression of TLR-4 (an LPS receptor) on monocytes of HD patients may reduce the induction of subsequent immune reactions. Reduced TLR-4 expression may thus be associated with a compromised immune function and high susceptibility to infection in HD patients. It has been difficult to identify the factors responsible for reduction of TLR-4 expression. However, since HD patients are exposed to one factor (ET contained in the dialysate) from the dialyzer, it seems possible that chronic stimulation with ET suppresses the expression of TLR-4 in HD patients. Although various reports have been published concerning whether the ET contained in dialysate can pass through the dialyzer (3-5), it seems likely that the ET in the dialysate passes through the membrane and acts on peripheral blood monocytes, resulting in reduction of TLR-4 expression, given the recent frequent use of large-pore-size membranes for dialysis. Furthermore, it has been shown that 
even if ET cannot pass through the membrane, it is adsorbed on the dialyzer, enabling it to act on peripheral blood monocytes and induce the formation of inflammatory cytokines (4). It thus appears that the ET contained in the dialysate reduces TLR-4 expression and thus compromises innate immunity in dialysis patients. Furthermore, since prolongation of dialysis results in significantly more reduction of TLR-4 expression, it is likely that innate immunity is compromised to a greater extent as the duration of exposure to ET contained in the dialysate becomes longer.

On the other hand, no such tendency was observed in the expression of TLR-2 (a receptor for PG) among HD patients. Instead, TLR-2 expression tended to decrease in those PD patients who were more likely to be exposed to PG. This suggests that compromised innate immunity in PD patients is due to exposure to PG (i.e. gram-positive bacteria) rather than to ET. However, since peritonitis is sometimes attributable to gram-negative bacteria, it seems probable that TLR-4 expression also tends to be decreased in PD patients.

Concerning the changes in TLR-2 and TLR-4 expression on peripheral blood monocytes following stimulation with LPS (ET), a previous study using intraperitoneal macrophages of mice demonstrated a reduction in TLR-4 expression following incubation in the presence of LPS, as well as reduction in the production of cytokines (27). In addition, crosstalk was observed between TLR-2 and TLR-4, and stimulation of TLR-4 increased the expression of TLR-2 $(28,29)$. In the present study as well, changes in TLR-2 and TLR-4 expression following stimulation with LPS were examined.

There was no significant difference in the time course of expression of these two molecules between the healthy and HD groups. In both groups, stimulation with LPS increased expression of TLR-2 and reduced expression of TLR-4. The magnitude of changes in expression of TLR-2 and TLR-4 tended to be smaller in PS membrane users and patients undergoing HD for a longer period of time. Thus, prolongation of dialysis and use of a PS membrane (which is more likely to result in exposure to microbial components such as ET) reduced the reactivity of TLR, suggesting that host-defensive reactions will be weaker in these patients.

In conclusion, suppression of expression of TLR-4 (an ET receptor involved in innate immunity) was noted in patients with renal failure, primarily those undergoing HD. TLR-4 expression was significantly and more markedly reduced as the duration of dialysis became longer. Furthermore, protection from new infection also appeared to be compromised in these patients. Both of these abnormalities appeared to be due to ET, a microbial component contaminating the dialysate.

\section{Acknowledgements}

The present study was supported by The Osaka Medical Research Foundation for Incurable Disease.

\section{References}

1. Shinzato T, Nakai S, Akiba T, Yamagami S, Yamazaki C, Kitaoka T, Kubo K, Maeda K and Morii H: Report of the annual statistical survey of the Japanese Society for Dialysis Therapy in 1996. Kidney Int 55: 700-712, 1999.
2. Andrew OT, Schoenfeld PY, Hopewell PC and Humphreys MH: Tuberculosis in patients with end-stage renal disease. Am J Med 68: 59-65, 1980 .

3. Tsuchida K, Takemoto Y, Yamagami S, Edney H, Niwa M, Tsuchiya M, Kishimoto T and Shaldon S: Detection of peptidoglycan and endotoxin in dialysate, using silkworm larvae plasma and limulus amebocyte lysate methods. Nephron 75 : 438-443, 1997.

4. Tsuchida K, Nakatani T, Sugimura K, Yoshimura R, Matsuyama $M$ and Takemoto $Y$ : Biological reaction by mediating reaction of endotoxin adsorbed dialysis membrane An in vitro study. Artif Organs 28: 231-234, 2004.

5. Nakatani T, Tsuchida K, Sugimura K, Yoshimura R and Takemoto Y: Response of peripheral blood mononuclear cells in hemodialyzed patients against endotoxin and muramyldipeptide. Int J Mol Med 10: 469-472, 2002.

6. Stenvinkel P, Heimburger O, Lindholm B, Kaysen GA and Bergstrom J: Are there two types of malnutrition in chronic renal failure? Evidence for relationships between malnutrition, inflammation and atherosclerosis (MIA syndrome). Nephrol Dial Transplant 15: 953-960, 2000.

7. Lonnemann G and Koch KM: Beta(2)-microglobulin amyloidosis, effects of ultrapure dialysate and type of dialyzer membrane. J Am Soc Nephrol 13 (suppl 1): 72-77, 2002.

8. Schiffl H, Lang SM and Bergner A: Ultrapure dialysate reduces dose of recombinant human erythropoietin. Nephron 83: 278-279, 1999.

9. Medzhitov R and Janeway C Jr: Innate immunity. N Engl J Med 343: 338-344, 2000.

10. Hoshino K, Takeuchi O, Kawai T, Sanjo H, Ogawa T, Takeda Y, Takeda K and Akira S: Toll-like receptor 4 (TLR4)-deficient mice are hyporesponsive to lipopolysaccharide: evidence for TLR4 as the Lps gene product. J Immunol 162: 3749-3752, 1999.

11. Takeuchi O, Hoshino K, Kawai T, Sanjo H, Takada H, Ogawa T, Takeda K and Akira S: Differential roles of TLR2 and TLR4 in recognition of gram-negative and gram-positive bacterial cell wall components. Immunity 11: 443-451, 1999.

12. Miyake K: Toll-like receptors and their roles in defense responses against infection. Kansenshogaku Zasshi 77: 473-479, 2003.

13. Ganz T: Defensins and host defense. Science 286: 420-421, 1999.

14. Ozinsky A, Underhill DM, Fontenot JD, Hajjar AM, Smith KD, Wilson CB, Schroeder L and Aderem A: The repertoire for pattern recognition of pathogens by the innate immune system is defined by cooperation between toll-like receptors. Proc Natl Acad Sci USA 97:13766-13771, 2000.

15. Shimazu R, Akashi S, Ogata H, Nagai Y, Fukudome K, Miyake K and Kimoto M: MD-2, a molecule that confers lipopolysaccharide responsiveness on Toll-like receptor 4. J Exp Med 189: 1777-1782, 1999.

16. Nagai Y, Akashi S, Nagafuku M, Ogata M, Iwakura Y, Akira S, Kitamura T and Kosugi A: Essential role of MD-2 in LPS responsiveness and TLR4 distribution. Nat Immunol 3: 667-672, 2002.

17. Agnese DM, Calvano JE, Hahm SJ, Coyle SM, Corbett SA, Calvano SE and Lowry SF: Human toll-like receptor 4 mutations but not CD14 polymorphisms are associated with an increased risk of gram-negative infections. J Infect Dis 186: $1522-1525,2002$.

18. Lorenz E, Mira JP, Frees KL and Schwartz DA: Relevance of mutations in the TLR4 receptor in patients with gram-negative septic shock. Arch Intern Med 162: 1028-1032, 2002.

19. Echchannaoui H, Frei K, Schnell C, Leib SL, Zimmerli W and Landmann R: Toll-like receptor 2-deficient mice are highly susceptible to Streptococcus pneumoniae meningitis because of reduced bacterial clearing and enhanced inflammation. J Infect Dis 186: 798-806, 2002.

20. Lorenz E, Mira JP, Cornish KL, Arbour NC and Schwartz DA: A novel polymorphism in the toll-like receptor 2 gene and its potential association with staphylococcal infection. Infect Immun 68: 6398-6401, 2000.

21. Netea MG, Van Der Graaf CA, Vonk AG, Verschueren I, Van Der Meer JW and Kullberg BJ: The role of toll-like receptor (TLR) 2 and TLR4 in the host defense against disseminated candidiasis. J Infect Dis 185: 1483-1489, 2002.

22. Abel B, Thieblemont N, Quesniaux VJ, Brown N, Mpagi J, Miyake K, Bihl F and Ryffel B: Toll-like receptor 4 expression is required to control chronic mycobacterium tuberculosis infection in mice. J Immunol 169: 3155-3162, 2002. 
23. Kang TJ and Chae GT: Detection of Toll-like receptor 2 (TLR2) mutation in the lepromatous leprosy patients. FEMS Immunol Med Microbiol 31: 53-58, 2001

24. Kurz P, Kohler H, Meuer S, Hutteroth T and Meyer zum Buschenfelde KH: Impaired cellular immune responses in chronic renal failure: evidence for a $\mathrm{T}$ cell defect. Kidney Int 29: 1209-1214, 1986.

25. Smogorzewski M and Massry SG: Defects in B-cell function and metabolism in uremia: role of parathyroid hormone. Kidney Int 59 (suppl 78): 186-189, 2001.

26. Ikushima H, Nishida T, Takeda K, Ito T, Yasuda T, Yano M, Akira S and Matsuda H: Expression of Toll-like receptors 2 and 4 is downregulated after operation. Surgery 135: 376-385, 2004.
27. Nomura F, Akashi S, Sakao Y, Sato S, Kawai T, Matsumoto M, Nakanishi K, Kimoto M, Miyake K, Takeda K and Akira S: Cutting edge: endotoxin tolerance in mouse peritoneal macrophages correlates with downregulation of surface Tolllike receptor 4 expression. J Immunol 164: 3476-3479, 2000.

28. Latz E and Golenbock DT: Receptor 'cross talk' in innate immunity. J Clin Invest 112: 1136-1137, 2003.

29. Matsuguchi T, Musikacharoen T and Ogawa T: Gene expression of Toll-like receptor 2, but not Toll-like receptor 4, is induced by LPS and inflammatory cytokines in mouse macrophages. J Immunol 165: 5767-5772, 2000. 